\title{
La producción científica actual en Didáctica de la Lengua y la Literatura en revistas españolas
}

\author{
Dimitrinka G. NíkLEVA \\ Beatriz Cortina PÉRez \\ Universidad de Granada
}

Recibido: 6 junio 2012 / Aceptado: 7 marzo 2013

ISSN: $1697-7467$

\begin{abstract}
RESUMEN: Esta investigación consiste en un estudio bibliométrico que pretende analizar la producción científica en el ámbito de Didáctica de la Lengua y la Literatura en tres revistas especializadas españolas. El análisis de varios indicadores nos permitirá definir el panorama actual y las tendencias predominantes: número de artículos publicados, temática, idioma empleado, idioma investigado, periodicidad, método de investigación, número de autores, etc. Analizamos todos los artículos (193 en total) durante tres años -2009, 2010 y 2011- en tres revistas representativas para este ámbito: Didáctica. Lengua y Literatura, Lenguaje y textos y Porta Linguarum. Aplicamos un método estadístico cuantitativo, realizado con el programa SPSS (Statistical Product and Service Solutions).

Palabras clave: estudio bibliométrico, investigación en Didáctica de la Lengua y la Literatura, revistas especializadas, análisis cuantitativo.
\end{abstract} Current Scientific Production on Language and Literature Didactics in Spanish
Journals

\begin{abstract}
This research presents a bibliometric study aiming at analysing the scientific production in the area of Language and Literature Didactics in three specialised Spanish journals. The analysis of various indicators will allow us to define the current panorama and the predominant trends: number of published articles, thematic scope, language used, language researched, periodicity, research method, number of authors, etc. We analyse all papers (193 in total) during three years - 2009, 2010 and 2011- of three specialised and representative journal of this area: Didáctica. Lengua y Literatura, Lenguaje y textos and Porta Linguarum. The quantitative method of statistical analysis with the SPSS software (Statistical Product and Service Solutions) is used.

Keywords: bibliometric study, research on Language and Literature Didactics, specialised journals, quantitative analysis.
\end{abstract}

\section{INTRODUCCIÓN}

El estado actual de la investigación en Didáctica de la Lengua y la Literatura es un tema de mucho interés para docentes e investigadores del área. La evolución de los parámetros de publicación científica y las exigencias de la carrera académica en España han provocado cambios en las revistas científicas (Fernádez-Quijada, 2010: 553). Por ello, hay que conocer estas exigencias para poder adaptarse a los nuevos retos en los temas de investigación y mejorar los indicios de calidad. Sin embargo, consideramos que es un tema bastante desconocido. Se 
investiga, pero desconocemos la situación, las tendencias, las lagunas de la investigación en un marco amplio. Los metaanálisis sobre la producción científica son escasos y no actualizados. Esto nos ha llevado a realizar este estudio bibliométrico sobre el estado actual de la investigación en el ámbito de Didáctica de la Lengua y la Literatura en el contexto español para detectar las tendencias predominantes y así poder formular sugerencias en cuanto a las lagunas que se producen.

El análisis bibliométrico por su naturaleza metodológica es meramente descriptivo. Por ello, aplicamos un método de investigación cuantitativa, realizado con el programa SPSS (Statistical Package for Social Sciences) para estudiar la dependencia entre las variables analizadas.

Con la convicción de que las revistas especializadas del área de Didáctica de la Lengua y la Literatura son las que reflejan las tendencias que predominan en el panorama actual en este ámbito, nos hemos planteado contrastar tres de las revistas españolas más representativas del área durante los años 2009, 2010 y 2011 y analizar todos los artículos (193 en total) según varios indicadores de la producción científica. Las tres revistas seleccionadas son: Didáctica. Lengua y Literatura, Lenguaje y textos y Porta Linguarum.

\section{Marco teórico}

\subsection{Situación de las revistas especializadas en Didáctica de la Lengua y la Literatura en España}

Antes de profundizar en el diseño y los resultados de nuestro estudio, nos parece oportuno ampliar la información básica sobre las revistas especializadas en el ámbito de Didáctica de la Lengua y la Literatura, puesto que son el tipo de publicación que mejor refleja el estado actual de esta área, sobre todo en el caso de investigadores entre el profesorado universitario.

Las tres revistas seleccionadas para este estudio están en la base de datos de DICE (Difusión y Calidad Editorial de las Revistas Españolas de Humanidades y Ciencias Sociales y Jurídicas). Didáctica. Lengua y Literatura se incluye también en IN-RECS (Índice de impacto. Revistas españolas de Ciencias Sociales).

Lenguaje y textos está recogida en la base de datos de ISOC, cuyos productores son CINDOC (Centro de Información y Documentación Científica) y CSIC (España). Está indexada en ISOC. Cumple con 32 criterios Latindex. No tiene factor de impacto. Se edita por la editorial Horsori / Sedll (Sociedad Española de Didáctica de la Lengua y la Literatura). Es una revista semestral, impresa.

Las bases de datos que incluyen la revista Didáctica. Lengua y Literatura son: FRANCIS, LLBA, MLA, ISOC. El editor de la revista es la Universidad Complutense de Madrid, Facultad de Educación, Departamento de Didáctica de la Lengua y la Literatura. Cumple con 32 criterios Latindex. Es una revista anual que presenta las dos versiones -impresa y electrónica-.

Los que nos dedicamos a la investigación en este ámbito consideramos muy representativas revistas como Lenguaje y textos o Porta Linguarum, por ejemplo. Las dos están 
incluidas en DICE, pero no en IN-RECS. Lenguaje y textos es la revista de la Sociedad Española de Didáctica de la Lengua y la Literatura (SEDLL). Porta Linguarum es una revista especializada en la didáctica de las lenguas extranjeras, editada por la Facultad de Ciencias de la Educación de la Universidad de Granada.Está incluida en el JCR (SSCI) y tiene factor de impacto. Es la revista con mejor posición de las tres seleccionadas. Está recogida en DICE, pero no en IN-RECS, a pesar de que su área de conocimiento es Didáctica de la Lengua y la Literatura y Lingüistica general (área temática Lingüística, Filología y Literatura). Las bases de datos que la incluyen son: A\&HCI, MLA, SSCI, ISOC. Tiene 32 criterios Latindex cumplidos. Entre las tres revistas, Porta Linguarum tiene el mayor índice de internacionalidad de las contribuciones: $42,86 \%$ frente a $28,57 \%$ de Didáctica. Lengua y Literatura y 2,94\% de Lenguaje y Textos.

En la tabla 1 sintetizamos la información sobre algunos indicios de calidad de las revistas (según DICE, última actualización de 28 de enero de 2011). A continuación, presentaremos también información sobre el factor de impacto y la posición de las revistas.

Tabla 1. Indicios de calidad de las revistas analizadas en el estudio

\begin{tabular}{|c|c|c|c|}
\hline Revista & $\begin{array}{c}\text { Criterios } \\
\text { Latindex }\end{array}$ & Bases de datos & $\begin{array}{c}\text { Internacionalidad } \\
\text { de las contribuciones }\end{array}$ \\
\hline Porta Linguarum & 32 & A\&HCI, SSCI, MLA, ISOC & 42,86 \\
\hline $\begin{array}{c}\text { Didáctica. Lengua y } \\
\text { Literatura }\end{array}$ & 32 & FRANCIS, LLBA, MLA, ISOC & 28,57 \\
\hline Lenguaje y textos & 32 & ISOC & 2,94 \\
\hline
\end{tabular}

Veamos la situación general de las revistas del área en la plataforma de DICE y de IN-RECS. Si realizamos la siguiente búsqueda en su web "área temática Ciencias de la Educación y área de conocimiento Didáctica de la Lengua y la Literatura", obtenemos catorce revistas que podemos clasificar solo por el número de criterios Latindex cumplidos (según la última actualización de DICE del 29 de julio de 2010). Lamentablemente, ninguna de estas catorce revistas está en la plataforma del ISI (Institute for Scientific Information) ni en la base de datos del SSCI (Social Science Citation Index).

Si un investigador de nuestra área quiere publicar en revistas con mejor posición, puede elegir entre las revistas incluidas en IN-RECS donde, según la última actualización en 2010, en el área temática de Educación están incluidas 72 revistas. El último año con índice de impacto calculado es 2010. Aquí están recogidas todas las áreas de conocimiento dentro del área temática de Educación, por lo que las revistas dedicadas a la Didáctica de la Lengua y la Literatura son solo cuatro. La primera de la lista por orden de índice de impacto que corresponde a nuestra área de investigación es Articles de Didactica de la Llengua i de la Literatura. Ocupa la posición $\mathrm{n}^{0} 39$ (tercer cuartil), con un factor de impacto de 0.065 en 2010. Hay que advertir que se publica principalmente en catalán lo que dificulta el acceso de muchos investigadores en España. La segunda revista del ámbito en IN-RECS es Textos de Didáctica de la Lengua y de la Literatura: posición $\mathrm{n}^{\circ} 50$, tercer cuartil y un factor de impacto de 0.048 en 2010. La tercera revista es MarcoELE. Revista de Didáctica: $\mathrm{n}^{\circ} 61$ de 72 (cuarto cuartil), con un factor de impacto de 0,020. Esta revista se recoge en IN-RECS 
por primera vez en 2010. La cuarta revista y la última del ámbito en IN-RECS es Didáctica. Lengua y Literatura; está en la posición $\mathrm{n}^{\circ} 72$ de 72 , en el tercer cuartil y sin factor de impacto en 2010.

En la tabla 2 recogemos el factor de impacto de las revistas y su posición entre 2008 y 2010, puesto que todavía no está calculado para 2011. Para Porta Linguarum fue calculado por primera vez en 2010 en ISI: JCR (SSCI). Para Didáctica. Lengua y Literatura presentamos los datos recogidos en IN-RECS. Añadimos los mismos datos para la revista Textos de Didáctica de la Lengua y la Literatura y MarcoELE. Revista de Didáctica para ampliar la comparación. La revista Lenguaje y Textos no está recogida en estas bases de datos, pero aparece en DICE, sin tener factor de impacto.

Tabla 2. Factor de impacto y posición de la revistas

\begin{tabular}{|c|c|c|c|c|c|c|}
\hline & \multicolumn{2}{|c|}{2008} & \multicolumn{2}{|c|}{2009} & \multicolumn{2}{|c|}{2010} \\
\hline & $\begin{array}{l}\text { factor de } \\
\text { impacto }\end{array}$ & posición & $\begin{array}{l}\text { factor de } \\
\text { impacto }\end{array}$ & posición & $\begin{array}{l}\text { factor de } \\
\text { impacto }\end{array}$ & posición \\
\hline $\begin{array}{l}\text { Porta Linguarum } \\
\text { (en JCR: SSCI) } \\
\text { en Linguistics } \\
\text { en Education \& } \\
\text { Educational Research }\end{array}$ & - & - & - & - & 0,160 & $\begin{array}{l}115 / 144 \\
166 / 184\end{array}$ \\
\hline $\begin{array}{l}\text { MarcoELE. Revista de } \\
\text { didáctica. } \\
\text { (en IN-RECS) }\end{array}$ & - & - & - & - & 0,020 & $\begin{array}{c}61 / 72 \\
4^{\circ} \text { cuartil }\end{array}$ \\
\hline $\begin{array}{l}\text { Textos de Didáctica } \\
\text { de la Lengua y la } \\
\text { Literatura } \\
\text { (en IN-RECS) }\end{array}$ & - & $\begin{array}{c}34 / 86 \\
2^{\circ} \text { cuartil }\end{array}$ & 0,037 & $\begin{array}{c}60 / 89 \\
3 \text { cuartil }\end{array}$ & 0,048 & $\begin{array}{c}50 / 72 \\
3 \text { cuartil }\end{array}$ \\
\hline $\begin{array}{l}\text { Didáctica. Lengua y } \\
\text { Literatura } \\
\text { (en IN-RECS) }\end{array}$ & 0 & $\begin{array}{c}86 / 86 \\
4^{\circ} \text { cuartil }\end{array}$ & 0,038 & $\begin{array}{c}54 / 89 \\
3 \text { cuartil }\end{array}$ & 0 & $\begin{array}{c}72 / 72 \\
4^{\circ} \text { cuartil }\end{array}$ \\
\hline
\end{tabular}

No nos proponemos mencionar todas las revistas existentes en nuestra área de investigación, puesto que la información es accesible en las fuentes que hemos citado anteriormente. Sin embargo, hemos querido resaltar algunos datos que quizás dificultan la posibilidad de seleccionar una revista con buena posición y especializada en el área de Didáctica de la Lengua y la Literatura.

Llama la atención el hecho de que no se observa una equivalencia entre la información que encontramos en DICE y en IN-RECS ni consideramos que la tiene que haber, pero sí sorprende que revistas con muy buena posición en DICE no estén recogidas en IN-RECS. Pongamos de ejemplo Porta Linguarum, Ocnos. Revista de estudios sobre lectura $\left(\mathrm{n}^{\circ} 1\right.$ en DICE) o Tejuelo. Didáctica de la Lengua y la Literatura $\left(\mathrm{n}^{\circ} 2\right)$. 
En cuanto a la revista Porta Linguarum, puesto que es la revista con mejor posición entre las analizadas en esta investigación, consideramos conveniente añadir más datos, según la plataforma ISI Web of Knowledge, de Thomson Reuters (2012). En la tabla 3 presentamos el número de citas por año.

Tabla 3. Citas de la revista Porta Linguarum por año

\begin{tabular}{|c|c|c|c|c|c|c|c|c|c|}
\hline 2001 & 2002 & 2003 & 2004 & 2005 & 2006 & 2007 & 2008 & 2009 & 2010 \\
\hline 0 & 0 & 0 & 4 & 5 & 3 & 4 & 4 & 4 & 0 \\
\hline
\end{tabular}

En la tabla 4 se recoge la contribución de las autocitas al cálculo del factor de impacto de Porta Linguarum.

Tabla 4. Contribución de las autocitas al cálculo del factor de impacto de Porta Linguarum

\begin{tabular}{|l|r|}
\hline Total citas & 24 \\
\hline Citas usadas en el cálculo del factor de impacto & 8 \\
\hline Factor de impacto & 0,160 \\
\hline Autocitas & $20(83 \%$ de 24$)$ \\
\hline Autocitas durante los años usados en el cálculo del factor de impacto & $7(87 \%$ de 8$)$ \\
\hline Factor de impacto sin las autocitas & 0,020 \\
\hline
\end{tabular}

Estamos convencidos de que los datos expuestos anteriormente reflejan parte de la compleja realidad relacionada con el tema de investigación en Didáctica de la Lengua y la Literatura. Si la relación entre la investigación y la posición de la revista (por los indicios de calidad) es de causa-efecto, conviene estudiar el estado actual de la investigación en este ámbito para hacer una evaluación de diagnóstico y poder proponer algunas vías de mejora.

\subsection{Objeto de la investigación en Didáctica de la Lengua y la Literatura}

En el marco teórico de este estudio cabe reflexionar sobre el objeto de la investigación en Didáctica de la Lengua y la Literatura. El estudio de Ana Camps (2004), titulado Objeto, modalidades y ámbitos de la investigación en Didáctica de la Lengua, es un estudio teórico del que nos parece conveniente resumir la opinión de la autora respecto al objeto de la investigación en el ámbito estudiado.

Ana Camps (2004: 12) hace hincapié en la relación entre investigación y docencia.

El objetivo del conocimiento didáctico no es simplemente descriptivo, sino que intenta interpretar y comprender la realidad de la enseñanza y aprendizaje de la lengua. Esta comprensión no es un fin en ella misma, sino que se orienta a la transformación de la práctica. La didáctica de la lengua es una disciplina de intervención: sus resultados han de conducir a mejorar el aprendizaje de la lengua y sus usos. 
Pero la investigación en didáctica de la lengua no tiene únicamente una función praxeológica, sino que aspira también a la creación de un cuerpo teórico de conocimientos. Este saber teórico tiene su origen en el análisis de la práctica, con la intención de darle sentido. Desde este punto de vista, la relación entre teoría y práctica no es una relación externa, sino intrínseca al conocimiento didáctico, y no hay prioridad entre los dos ámbitos, sino que son dos componentes de una misma actividad humana que es la de enseñar y aprender [...].

Estas reflexiones llevan a plantear la pregunta de cómo surge la investigación teórica a partir de la práctica. Siguiendo a varios autores, Ana Camps distingue cinco niveles en dos bloques en el proceso de reflexión a partir de la práctica: uno de reflexión durante la acción y un segundo nivel de reflexión sobre la acción, una vez finalizada.

- La reflexión durante la acción se circunscribe al desarrollo de las actividades y está estrechamente unida a la práctica. Consta de dos niveles:

- Reacción inmediata ante una situación relativamente problemática.

- Readaptación de la actuación planificada.

- La reflexión sobre la acción se produce en tres niveles:

- Reflexión después de la actuación. Puede ocasionar la modificación de la planificación.

- Recogida de información, análisis de los datos y evaluación. En este nivel es muy importante la validez y la fiabilidad de la investigación.

- En este último nivel el análisis lleva a una lectura crítica de las teorías subyacentes a la actuación y se produce una revisión de las teorías existentes.

La síntesis que ofrece Camps (2004) destaca que el objetivo de la investigación en Didáctica de la Lengua es elaborar conocimiento teórico para poder intervenir y mejorar la práctica. Hay que recordar también que los procedimientos para llevar a cabo la investigación son diversos y complementarios.

\subsection{Una duda sobre la relación entre investigación y docencia}

Muchos investigadores opinan que existe una estrecha relación entre investigación y docencia, mientras que Ronald Barnett (2008, citado por Bausela, 2010) plantea muchas dudas al respecto en su libro titulado Para una transformación de la universidad. Nuevas relaciones entre investigación, saber y docencia. El autor pone de manifiesto que no se conoce la naturaleza de la conexión entre investigación y docencia, ni siquiera se afirma la existencia de esta conexión. Según Bausela (2010) en su recensión del libro, el autor considera que existe una abundante bibliografía de gran solidez retórica, pero a la vez señala una pobre documentación empírica sobre esta relación.

Sin embargo, R. Barnett sí considera que el saber pedagógico puede tender un puente entre la investigación y la docencia. Destaca la importancia de las tesis doctorales en este sentido, puesto que demuestran el aprendizaje en un modo de investigación (Bausela, 2010).

En el libro se discute otro tema problemático para los profesores universitarios: la incompatibilidad entre la investigación y la docencia y la dificultad de conseguir el equilibrio necesario, teniendo en cuenta que los profesores se seleccionan para enseñar, pero se les 
evalúa y promociona por investigar (Vidal y Quintanilla, 2000 y Sancho, 2001). Por todo ello, destacan la necesidad de proporcionar una formación adecuada al profesorado universitario que le permita adaptarse al Espacio Europeo de Educación Superior.

\subsection{Estudios precedentes}

Entre las investigaciones precedentes sobre el tema estudiado hemos optado por destacar los de Alario (1998), Battaner (2002), Vez (2009) y Níkleva y Cortina (2011).

El estudio de Alario Trigueros y otros (1998) se titula El desarrollo de Didáctica de la Lengua y la Literatura: consideraciones sobre su consolidación. El objeto del estudio son las comunicaciones presentadas en las primeras seis reuniones científicas (congresos y simposios) organizadas por la Sociedad Española de Didáctica de la Lengua y la Literatura. Las variables estudiadas son: autor, título, congreso, localidad, procedencia (nivel educativo del que proceden los autores), campo (lengua materna L1 o lengua extranjera o segunda lengua L2), tipos (experiencia, propuesta, teoría, investigación), ámbito (de aplicación, nivel educativo), temas, lengua, observaciones. Hacen hincapié en la temática abordada lo que permite determinar las tendencias más significativas. Los años estudiados son seis: desde 1989 hasta 1994, inclusive. Una de las conclusiones principales de los autores de este estudio es que las comunicaciones que suponen una investigación rigurosa representan un porcentaje muy bajo (14\% de los primeros tres años), por lo que hay que promover la investigación y establecer unos medios adecuados para su difusión.

En 2002 María Paz Battaner Arias publica un estudio sobre la Investigación en "Didáctica de la lengua y la literatura" y la consolidación del Área de Conocimiento (Battaner, 2002). La investigación pretende abarcar todo lo que se ha hecho público desde la creación de los departamentos de didácticas específicas. Su investigación se subdivide en cinco apartados:

- Adquisición de la sintaxis y el discurso.

- Recogida y análisis de datos sobre competencias.

- Recogida y análisis de datos de fenómenos en el proceso de enseñanza-aprendizaje.

- Estudios históricos sobre el desarrollo de la disciplina.

- Líneas experimentales y de intervención.

Nos gustaría señalar también el estudio de Fernández Cano y Bueno Sánchez (1998), a pesar de que no trata sobre el área de Didáctica de la Lengua y la Literatura, pero representa una síntesis de los estudios bibliométricos españoles en Educación. Según las conclusiones de estos autores, el instrumento más usado en la investigación es la encuesta por cuestionario. Los métodos cuantitativos superan abrumadoramente a los cualitativos. Los estudios experimentales son escasos y faltan por completo los estudios longitudinales.

Hay que destacar también un metaestudio de José Manuel Vez Jeremías (2009) sobre la Didáctica de las Lenguas-Culturas en la Comunidad Autónoma de Galicia en el que se analizan los productos de investigación de tres universidades gallegas y de centros educativos de niveles previos a la Universidad. El estudio ha sido publicado en 2009, pero abarca el período de 1989 a 2001. Las líneas de investigación se distribuyen en cinco bloques y los tipos de producto de investigación corresponden a la siguiente clasificación: 
- Publicaciones (libros, capítulos de libros, artículos).

- Proyectos de investigación.

- Tesis doctorales.

- Organización de congresos, seminarios u otros eventos científicos.

En su estudio Vez refleja la opinión de la comunidad científica (española e internacional) que no otorga a nuestro ámbito de investigación el suficiente rigor científico, algo que se considera un hecho, según Mackey en los años 60 (1965). Obviamente, ha transcurrido mucho tiempo y nuestro ámbito de investigación ha incorporado métodos de investigación que quizás puedan mejorar la consideración anteriormente citada. No se puede negar la influencia que han ejercido el ámbito francófono y, más tarde, el anglosajón sobre la investigación en España.

El paradigma de la investigación sobre la enseñanza que imperaba en las últimas décadas del siglo XX correspondía a un modelo de investigación proceso-producto. Se llevaba a cabo desde el exterior del aula, pero se centraba en lo que ocurría dentro del aula (Vez, 2009: 28): procesos de enseñanza, comportamientos de alumnos y profesor, análisis de errores, análisis del discurso, estrategias de interacción lingüística, etc. Recientemente se ha producido un cambio que ha llevado al desarrollo de otro paradigma de investigación que aplica una metodología cualitativa y/o cuantitativa desde el interior del aula, desde la perspectiva de los propios agentes en el proceso educativo.

Consideramos importante resaltar una idea más de este autor que compartimos plenamente: las ideas de innovación en nuestro ámbito no deben producirse entre investigadores exclusivamente universitarios, sino apostar por la colaboración con docentes de niveles previos a la universidad. Por otra parte, conviene también mejorar la colaboración entre distintas universidades que, según Vez, en algunas comunidades autónomas (Cataluña, Andalucía) está más conseguida que en otras (Galicia, por ejemplo).

De los resultados del estudio de Vez optamos por incluir el que se refiere a los artículos, puesto que nuestro estudio recoge solo artículos: 35 de los 82 artículos en total se centran en líneas de investigación exclusivamente dedicadas a los diversos aspectos de la didáctica de una lengua extranjera.

Por último, Níkleva y Cortina ofrecen un estudio actual sobre las Tendencias en la investigación actual en Didáctica de la Lengua y la Literatura (2011), en el que analizan la producción científica en dos revistas españolas durante dos años ( 78 artículos en total, durante 2009 y 2010). En el presente estudio nos proponemos ampliar la investigación en cuanto al período (tres años), el número de revistas (tres) y, por consiguiente, de artículos analizados (193). Además, ofrecemos un marco teórico mucho más amplio que incluye los estudios precedentes y profundizamos en el análisis de los indicios de calidad de las revistas.

\subsection{Programas informáticos utilizados en la investigación sobre Didáctica de la Lengua y la Literatura}

En los últimos años se observa un aumento de la aplicación de métodos cuantitativos y cualitativos en el análisis de datos estadísticos en el área de Didáctica de la Lengua y la Literatura.

Para el análisis cuantitativo el programa más usado es el SPSS (Statistical Product and Service Solutions). En el análisis cualitativo quizás predomina el uso de Nudist (NVIVO). 
Para el análisis de texto y del léxico recomendaríamos otras dos herramientas (Escobar y Sánchez, 2009). Advanced Text Analyser (ATA) es una herramienta para analizar el contenido del texto y clasificar las palabras según diferentes criterios. Proporciona también el número total de palabras y de oraciones.

$P$-lex es un programa informático que calcula el índice de sofisticación del texto, basándose en la proporción de palabras "fáciles" y "difíciles" que contiene. Clasifica las palabras en cuatro categorías, según la frecuencia de uso.

Las nuevas tecnologías ofrecen un número cada vez mayor de herramientas útiles para la investigación, por lo que conviene conocerlos y aprovecharlos en los análisis.

\section{OBJETIVOS E HiPótesis de LA INVESTIGACIÓN}

Hemos diseñado la investigación con los siguientes objetivos:

1. Estudiar la temática de investigación en el área de Didáctica de la Lengua y la Literatura y su frecuencia.

2. Comprobar si se investiga más sobre Lengua o sobre Literatura.

3. Comprobar qué etapa educativa predomina en las investigaciones.

4. Identificar los métodos de investigación que se utilizan.

5. Investigar sobre la modalidad de la lengua que predomina (oral o escrita).

6. Estudiar la relación entre la revista y el método de investigación.

7. Establecer la relación entre la posición de la revista según los indicios de calidad y los métodos de investigación de los artículos publicados.

En la etapa inicial formulamos las siguientes hipótesis:

1. Las habilidades lingüísticas son el tema que predomina.

2. Se investiga más sobre Lengua y menos sobre Literatura.

3. Predomina la investigación del tipo teórico sin análisis de datos.

4. La modalidad escrita de la lengua predomina frente a la modalidad oral.

5. Predomina la investigación teórica y los artículos en formato de propuesta didáctica o experiencia didáctica.

6. Las revistas con mejor posición en cuanto a los indicios de calidad publican con mayor frecuencia artículos de investigación cuantitativa y cualitativa frente a la investigación teórica que representa una revisión bibliográfica sin análisis de datos.

\section{Metodología}

\subsection{Muestra de datos}

Para investigar el estado actual de este tema nos hemos planteado realizar un estudio bibliométrico de algunas revistas españolas indexadas que consideramos representativas en el ámbito de Didáctica de la Lengua y la Literatura. Como hemos señalado anteriormente, hemos seleccionado tres revistas: 1) Didáctica. Lengua y Literatura, 2) Lenguaje y textos y 
3) Porta Linguarum. Los criterios de selección, tratados en profundidad en el apartado 2.1., son tres: área de conocimiento de Didáctica de la Lengua y la Literatura, lenguas: español, inglés y francés; indexación en bases de datos: DICE, IN-RECS y JCR (SSCI).

Presentamos un metaanálisis de todos los artículos publicados (193) en estas tres revistas durante los años 2009, 2010 y 2011. La tabla 5 contiene el número de artículos por revista y la periodicidad de la revista.

Tabla 5. Número de artículos por revista y periodicidad

\begin{tabular}{|c|c|c|}
\hline REVISTA & NÚMERO DE ARTÍCULOS & $\begin{array}{c}\text { PERIODICIDAD } \\
\text { números por año }\end{array}$ \\
\hline Didáctica. Lengua y literatura & $48(24,9 \%)$ & 1 \\
\hline Lenguaje y textos & $71(36,8 \%)$ & 2 \\
\hline Porta Linguarum & $74(38,3 \%)$ & 2 \\
\hline & Total: 193 & \\
\hline
\end{tabular}

\subsection{Variables y método de análisis}

Para nuestro estudio hemos aplicado un método de análisis estadístico cuantitativo, realizado con el programa estadístico Statistical Product and Service Solutions (SPSS), el más usado en Ciencias Sociales para el análisis de datos cuantitativos.

Con nuestra investigación pretendemos estudiar las tendencias predominantes en la investigación en Didáctica de la Lengua y la Literatura. Para ello hemos usado las siguientes variables cualitativas:

1) revista

2) año

3) en qué lengua está escrito el artículo (español, inglés, francés, otro idioma)

4) sobre qué lengua o literatura se investiga (español, inglés, francés, otro idioma)

5) subárea: L1 (lengua materna), LE (lengua extranjera) o L2 (segunda lengua), Literatura, varias de las anteriores

6) etapa educativa (Infantil, Primaria, Secundaria y Bachillerato, Universidad, sin especificar o varias de las anteriores)

7) temática:

- habilidades lingüísticas

- sintaxis

- morfología

- fonética

- competencias

- evaluación

- bilingüismo y plurilingüismo; interculturalidad y multiculturalidad; alumnado inmigrante

- léxico

- nuevas tecnologías (TIC) 
- ortografía

- análisis de errores

- didáctica general (teoría general, modelos, enfoques, aprendizaje, etc.)

- comentario de textos

- libros de texto (análisis de libros de texto, etc.)

- literatura

- pragmática, lingüística del texto y análisis del discurso

- EEES (Espacio Europeo de Educación Superior: créditos ECTS, tutorías, etc.)

- otro tema

8) modalidad de la lengua (oral, escrita, sin especificar)

9) sexo de los autores (hombre, mujer, mixto en el caso de varios autores)

10) número de los autores (uno, dos, más de dos)

11) método de investigación (formato del artículo)

- investigación teórica (revisión bibliográfica, sin análisis de datos)

- métodos cualitativos

- métodos cuantitativos

- métodos mixtos (cuantitativos y cualitativos)

- propuesta didáctica o experiencia didáctica

- otro método

Con estas variables se ha realizado en primer lugar un análisis descriptivo para conocer las frecuencias y los porcentajes de cada una y, a continuación, un análisis inferencial para estudiar la relación de dependencia entre las variables.

\section{Análisis y discusión de los Resultados}

A continuación se presentan los resultados de los análisis estadísticos realizados según los objetivos que nos hemos planteado y las hipótesis que hemos formulado.

\subsection{Resultados del análisis descriptivo}

Nuestro primer objetivo pretendía estudiar la temática de la investigación en el área de Didáctica de la Lengua y la Literatura.

El análisis descriptivo de frecuencia nos proporciona el siguiente resultado que confirma nuestra hipótesis (fig. 1): predomina el tema de habilidades lingüísticas (expresión oral y escrita; comprensión oral y escrita), seguido por el tema de Literatura, pero tenemos que advertir que la literatura como tema se solapa con uno de los subáreas que hemos formulado. Además, si todos los demás temas son de Lengua, influye el hecho de que los temas de Literatura se presentan sin ser desglosados en subtemas. En tercer lugar están los temas de didáctica general.

El tema con menor presencia es el de sintaxis. La investigación sobre temas dedicados a la ortografía, al análisis de errores o al Espacio Europeo de Educación Superior también es escasa.

Dentro de la temática, hemos englobado en "otro" temas como: la enseñanza del español como lengua extranjera con fines específicos, la traducción, los refranes, la historia de 
la enseñanza de idiomas, la influencia de las estancias en el extranjero para el aprendizaje de una lengua extranjera, etc.

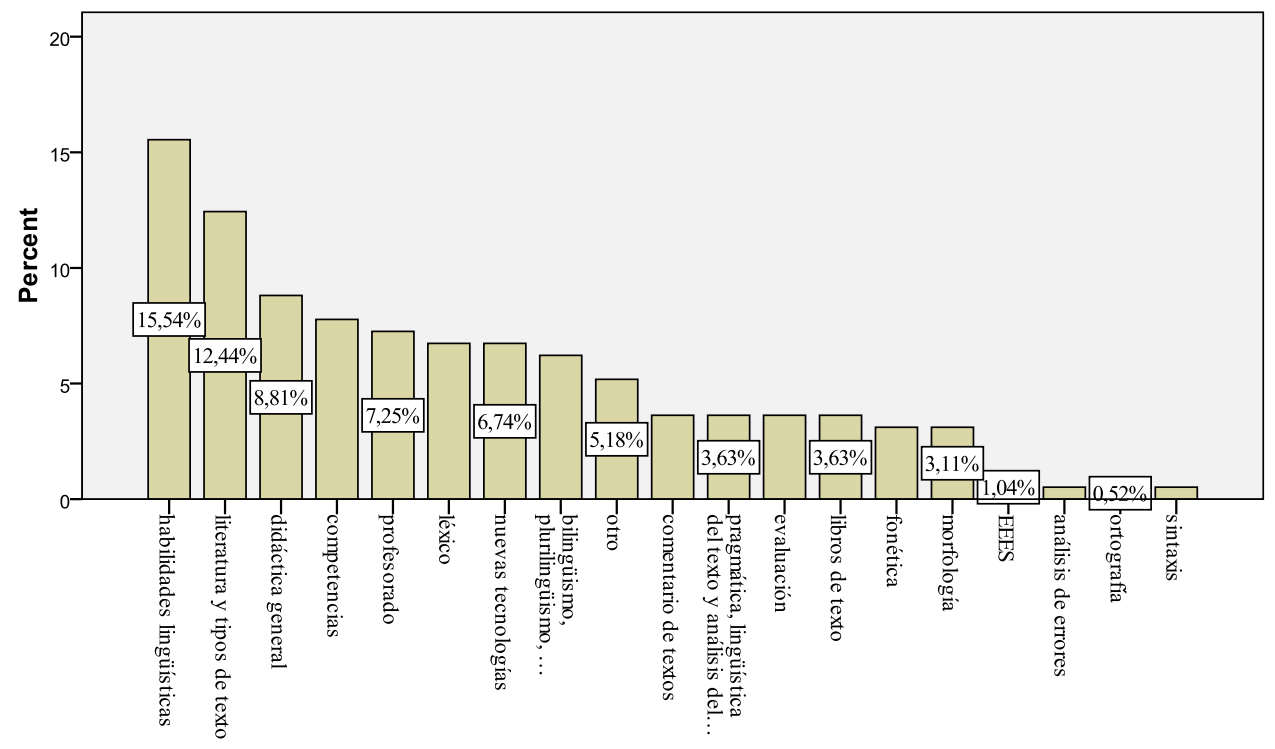

Fig. 1. Temática y su frecuencia (por porcentaje, en orden descendente)

Con este gráfico confirmamos también nuestra segunda hipótesis: se investiga más sobre Lengua $(87,56 \%)$ que sobre Literatura $(12,44 \%)$.

Hemos agrupado todos los temas en cuatro subáreas:

1) L1 (lengua materna o nativa)

2) LE y L2 (lengua extranjera y segunda lengua)

3) Literatura

4) varias de las anteriores

Por orden descendiente se sitúan de la siguiente manera: LE y L2 (113 artículos, 58,5\%), L1 (39 artículos; 20,2\%), Literatura (21 artículos; 10,9\%) y varias de las subáreas $(19 ; 9,8 \%)$.

Uno de nuestros objetivos era comprobar sobre qué etapa educativa se investiga más. Los resultados se pueden observar en el siguiente gráfico (ordenados por orden descendiente). 


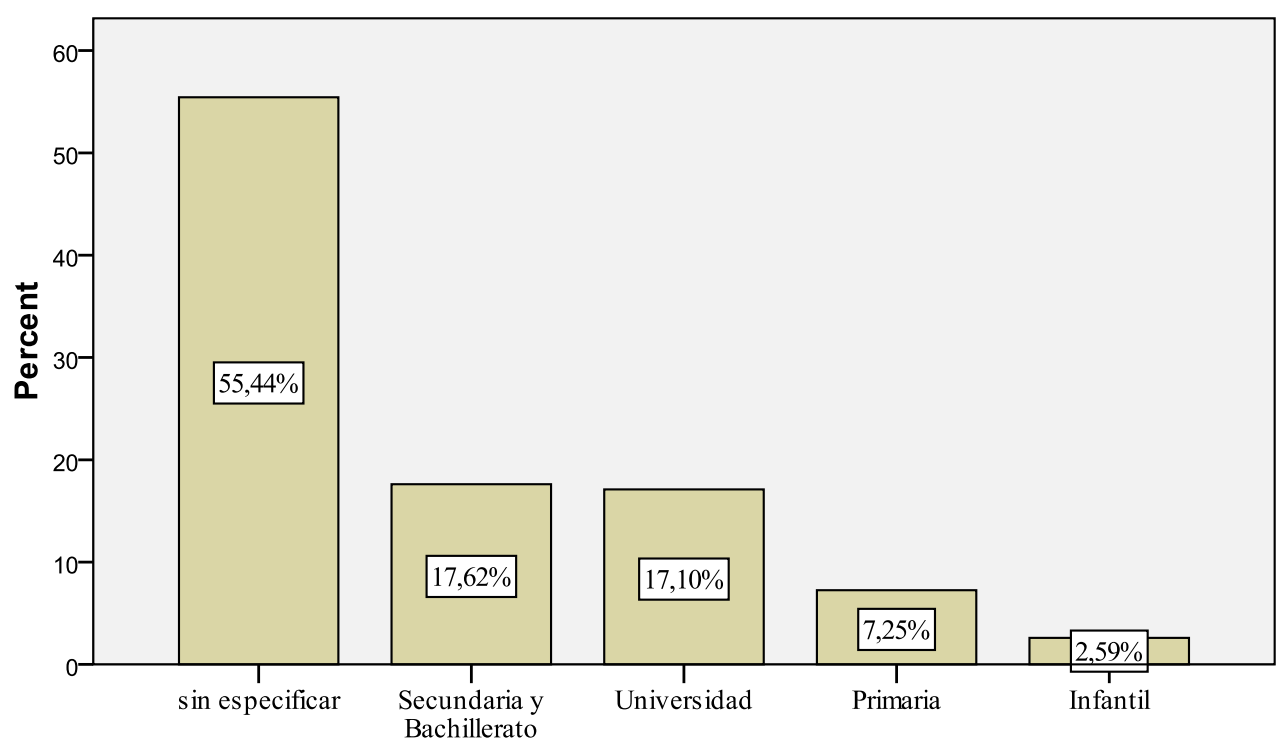

Fig. 2. Etapas educativas y su frecuencia en la investigación

Tenemos que advertir que muchas de las investigaciones no especifican una etapa en concreto o se refieren a varias a la vez (Infantil y Primaria o Primaria y Secundaria, etc.) lo que se refleja en el resultado del mayor porcentaje: $55,44 \%$.

Veamos qué etapa predomina en cada revista (tabla 6). El mayor porcentaje en las tres revistas corresponde a las investigaciones que no especifican la etapa. Sin embargo, en el resto se observa que en Didáctica. Lengua y Literatura y en Lenguaje y textos se publican más investigaciones sobre la etapa de la ESO y de Bachillerato, mientras que Porta Linguarum se centra más en la etapa de universidad.

Tabla 6. Etapas educativas y revistas

\begin{tabular}{|l|c|c|c|c|}
\hline & & \multicolumn{3}{|c|}{ Revista } \\
\hline \multirow{5}{*}{ Etapa educativa } & & $\begin{array}{c}\text { Didáctica. } \\
\text { Lengua } \\
\text { Literatura }\end{array}$ & Lenguaje y textos & Porta Linguarum \\
\hline \multirow{5}{*}{} & Infantil & 1 & 3 & 1 \\
\cline { 2 - 5 } & Primaria & 1 & 6 & 7 \\
\cline { 2 - 5 } & $\begin{array}{c}\text { Secundaria y } \\
\text { Bachillerato }\end{array}$ & 12 & 12 & 10 \\
\cline { 2 - 5 } & Universidad & 9 & 9 & 15 \\
\cline { 2 - 5 } & sin especificar & 25 & 25 & 41 \\
\hline & & & & \\
\hline
\end{tabular}


Nuestra hipótesis sobre los métodos de investigación que se aplican con más frecuencia en Didáctica de la Lengua y la Literatura se confirma también (fig. 3): todavía predominan las investigaciones de tipo teórico o revisión bibliográfica (42,49\%). En segundo lugar están los artículos elaborados como una propuesta didáctica o experiencia didáctica (24,87\%). Las investigaciones cuantitativas están en aumento en los últimos años y ocupan el tercer lugar en el corpus analizado $(19,17 \%)$.

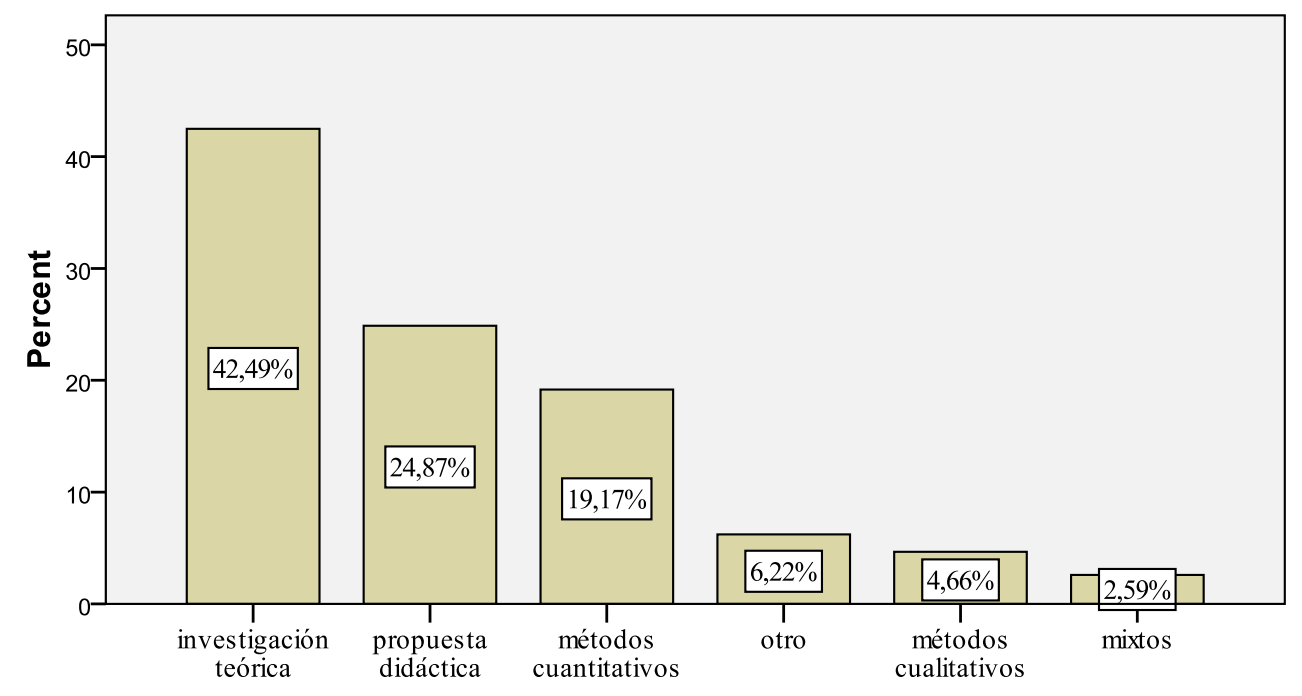

Fig. 3. Método de investigación

Se ha observado también que la investigación sobre la lengua escrita predomina frente a la oral. Es cierto que un alto porcentaje de los artículos no especifica la modalidad lingüística a la que se refiere $(55,96 \%)$, pero cuando se concreta, la modalidad escrita ocupa un $30,05 \%$ frente a un $13,95 \%$ de la modalidad oral.

\subsection{Resultados del análisis inferencial}

Hemos realizado el análisis inferencial mediante el test de independencia con la prueba de chi-cuadrado y el test exacto de Fisher para estudiar si hay relación de dependencia entre las variables.

Se ha confirmado la relación de dependencia en los siguientes casos:

1) Relación de dependencia entre la revista y el método de investigación.

Los resultados obtenidos confirman la relación de dependencia de grado medio entre la revista y el método de investigación: $x^{2}(10)=54.818, \mathrm{p}<.001, \mathrm{~V}$ de Cramer $=.377$. 7):

El resultado del método usado por revista nos proporciona los siguientes datos (tabla

En Porta Linguarum predomina la investigación con métodos cuantitativos (28 artículos de 74). En segundo lugar están los de investigación teórica (22 de 74). Llama la atención 
en comparación con las demás revistas que el formato de propuesta didáctica o experiencia en el aula no ocupa una posición privilegiada, sino que se sitúa en el cuarto lugar (7 artículos de 74).

En Didáctica. Lengua y literatura predomina la investigación teórica (24 de 48 artículos), seguida por los artículos con el formato de propuesta didáctica (18 de 48). La investigación con análisis estadístico de datos es escasa: 3 artículos que aplican los métodos cuantitativos y 1 artículo de investigación cualitativa.

En Lenguaje y textos la situación es parecida. La investigación teórica ocupa el primer lugar (36 de 71 artículos). A la propuesta didáctica le corresponde el segundo lugar (23 de 71). En cuanto a la investigación con análisis estadístico, la revista se sitúa en la posición intermedia entre las tres revistas. Cuenta con 6 artículos de investigación cuantitativa y 5 de investigación cualitativa.

Tabla 7. Métodos de investigación por revista

\begin{tabular}{|c|c|c|c|c|c|c|c|c|c|c|}
\hline & \multicolumn{9}{|c|}{ Revista } \\
\hline & & \multicolumn{3}{|c|}{ Didáctica. Lengua y Literatura } & \multicolumn{3}{|c|}{ Lenguaje y textos } & \multicolumn{3}{|c|}{ Porta Linguarum } \\
\hline & & $\begin{array}{l}\text { Núm. } \\
\text { artículos }\end{array}$ & $\begin{array}{c}\text { \% por } \\
\text { columna }\end{array}$ & $\begin{array}{l}\% \text { por } \\
\text { fila }\end{array}$ & $\begin{array}{l}\text { Núm. } \\
\text { artículos }\end{array}$ & $\begin{array}{c}\% \text { por } \\
\text { columna }\end{array}$ & $\begin{array}{l}\% \text { por } \\
\text { fila }\end{array}$ & $\begin{array}{c}\text { Núm. } \\
\text { artículos }\end{array}$ & $\begin{array}{c}\text { \% por } \\
\text { columna }\end{array}$ & $\begin{array}{c}\text { \% por } \\
\text { fila }\end{array}$ \\
\hline \multirow{6}{*}{ 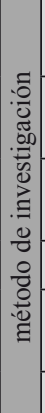 } & $\begin{array}{c}\text { investigación } \\
\text { teórica }\end{array}$ & 24 & $50,0 \%$ & $29,3 \%$ & 36 & $50,7 \%$ & $43,9 \%$ & 22 & $29,7 \%$ & $26,8 \%$ \\
\hline & $\begin{array}{c}\text { métodos } \\
\text { cuantitativos }\end{array}$ & 3 & $6,3 \%$ & $8,1 \%$ & 6 & $8,5 \%$ & $16,2 \%$ & 28 & $37,8 \%$ & $75,7 \%$ \\
\hline & $\begin{array}{c}\text { métodos } \\
\text { cualitativos }\end{array}$ & 1 & $2,1 \%$ & $11,1 \%$ & 5 & $7,0 \%$ & $55,6 \%$ & 3 & $4,1 \%$ & $33,3 \%$ \\
\hline & mixtos & 0 &, $0 \%$ &, $0 \%$ & 1 & $1,4 \%$ & $20,0 \%$ & 4 & $5,4 \%$ & $80,0 \%$ \\
\hline & $\begin{array}{l}\text { propuesta } \\
\text { didáctica }\end{array}$ & 18 & $37,5 \%$ & $37,5 \%$ & 23 & $32,4 \%$ & $47,9 \%$ & 7 & $9,5 \%$ & $14,6 \%$ \\
\hline & otro & 2 & $4,2 \%$ & $16,7 \%$ & 0 &, $0 \%$ &, $0 \%$ & 10 & $13,5 \%$ & $83,30 \%$ \\
\hline
\end{tabular}

2) Relación de dependencia entre la revista y la lengua en la que se investiga.

Según los resultados, observamos que Porta Linguarum publica predominantemente en inglés (46 de 74 artículos), mientras que en las otras dos revistas predominan las publicaciones en español (43 de 48 en Didáctica. Lengua y Literatura y 60 de 71 en Lenguaje $y$ textos).

La relación de dependencia de grado medio entre estas dos variables se confirma por los valores de $\mathrm{V}$ de Cramer de .469 , chi-cuadrado $\left(x^{2}(6)=84,975, \mathrm{p}<.001\right.$ y del test exacto de Fisher $\mathrm{F}=.000$.

3) Relación de dependencia entre el método de investigación y el número de autores.

En el análisis descriptivo hemos observado que predominan los artículos con un autor (129 artículos; 66,8\%), seguidos por los de dos autores (49 artículos; $25,4 \%$ ) y en tercer lugar, más de dos autores (15 artículos; 7,8\%). 
Los valores del chi-cuadrado $\left(x^{2}(10)=22.701, \mathrm{p}<.05\right)$ y el test exacto de Fisher $(\mathrm{F}=0.005)$ confirman una dependencia de grado bajo (V de Cramer 0.243) entre estas dos variables: el trabajo en equipo favorece la investigación que utiliza métodos estadísticos (tabla 8).

Tabla 8. Relación entre el método de investigación y el número de autores

\begin{tabular}{|c|c|c|c|c|c|c|c|c|c|c|}
\hline & \multicolumn{9}{|c|}{ Número de autores } \\
\hline & & \multicolumn{3}{|c|}{ uno } & \multicolumn{3}{|c|}{ dos } & \multicolumn{3}{|c|}{ más de dos } \\
\hline & & $\begin{array}{c}\text { Núm. } \\
\text { artículos }\end{array}$ & $\begin{array}{c}\% \text { por } \\
\text { columna }\end{array}$ & $\begin{array}{c}\% \text { por } \\
\text { fila }\end{array}$ & $\begin{array}{c}\text { Núm. } \\
\text { artículos }\end{array}$ & $\begin{array}{c}\% \text { por } \\
\text { columna }\end{array}$ & $\begin{array}{c}\begin{array}{c}\% \text { por } \\
\text { fila }\end{array} \\
\end{array}$ & $\begin{array}{c}\text { Núm. } \\
\text { artículos }\end{array}$ & $\begin{array}{c}\begin{array}{c}\% \text { por } \\
\text { columna }\end{array} \\
\end{array}$ & $\begin{array}{c}\begin{array}{c}\% \text { por } \\
\text { fila }\end{array} \\
\end{array}$ \\
\hline \multirow{6}{*}{ 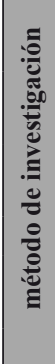 } & $\begin{array}{l}\text { investigación } \\
\text { teórica }\end{array}$ & 65 & $50,4 \%$ & $79,3 \%$ & 12 & $24,5 \%$ & $14,6 \%$ & 5 & $33,3 \%$ & $6,1 \%$ \\
\hline & $\begin{array}{l}\text { métodos } \\
\text { cuantitativos }\end{array}$ & 14 & $10,9 \%$ & $37,8 \%$ & 17 & $34,7 \%$ & $45,9 \%$ & 6 & $40,0 \%$ & $16,2 \%$ \\
\hline & $\begin{array}{l}\text { métodos } \\
\text { cualitativos }\end{array}$ & 6 & $4,7 \%$ & $66,7 \%$ & 2 & $4,1 \%$ & $22,2 \%$ & 1 & $6,7 \%$ & $11,1 \%$ \\
\hline & mixtos & 3 & $2,3 \%$ & $60,0 \%$ & 2 & $4,1 \%$ & $40,0 \%$ & 0 &, $0 \%$ &, $0 \%$ \\
\hline & $\begin{array}{l}\text { propuesta } \\
\text { didáctica }\end{array}$ & 32 & $24,8 \%$ & $66,7 \%$ & 14 & $28,6 \%$ & $29,2 \%$ & 2 & $13,3 \%$ & $4,2 \%$ \\
\hline & otro & 9 & $7,0 \%$ & $75,0 \%$ & 2 & $4,1 \%$ & $16,7 \%$ & 1 & $6,7 \%$ & $8,3 \%$ \\
\hline
\end{tabular}

En cuanto a la investigación teórica (un total de 82 artículos), un 79,3\% de los artículos se ha realizado por un autor; un $14,6 \%$, por dos autores y un $6,1 \%$, por más de dos autores.

En el caso de dos autores, predomina la investigación con métodos cuantitativos (34,7\%), seguida por el formato de propuesta didáctica $(28,6 \%)$, en tercer lugar, la investigación teórica $(24,5 \%)$, los métodos cualitativos $(4,1 \%)$ y los métodos mixtos $(4,1 \%)$.

4) La relación entre la revista y la lengua sobre la que se investiga.

Los valores de frecuencia del análisis descriptivo señalan que predomina la investigación sobre el español ( 82 artículos; 42,5\%), seguido por el inglés (68 artículos; 35,2\%). El francés es objeto de estudio de 4 artículos $(2,1 \%)$, mientras que los estudios que no especifican la lengua son $30(15,5 \%)$. Un idioma distinto de los anteriormente señalados ha sido objeto de estudio en 9 artículos $(4,7 \%)$.

Los datos de la relación entre la revista y la lengua sobre la que se investiga se pueden consultar en la tabla 9. En Porta Linguarum 49 de 74 artículos son investigaciones sobre el inglés. En Didáctica. Lengua y Literatura predomina la investigación sobre la lengua española (28 de 48 artículos). En Lenguaje y textos 40 de 71 artículos están dedicados a la lengua española.

Existe relación de dependencia entre las dos variables: $x^{2}(10)=60,309, \mathrm{p}<.001$. Del test exacto de Fisher obtenemos el valor de $\mathrm{F}=.000$. El valor de $\mathrm{V}$ de Cramer .395 indica una dependencia de grado medio. 
Tabla 9. Relación entre las variables revista y lengua sobre la que se investiga

\begin{tabular}{|c|c|c|c|c|c|c|c|}
\hline & \multicolumn{5}{|c|}{ sobre qué lengua se investiga } & \multirow[b]{2}{*}{ Total } \\
\hline & & español & inglés & francés & otro idioma & $\begin{array}{l}\text { cualquier } \\
\text { lengua }\end{array}$ & \\
\hline \multirow{3}{*}{ revista } & $\begin{array}{l}\text { Didáctica. } \\
\text { Lengua y } \\
\text { Literatura }\end{array}$ & $28(58,3 \%)$ & $11(22,9 \%)$ & $2(4,2 \%)$ & $2(4,2 \%)$ & $5(10,4 \%)$ & 48 \\
\hline & Lenguaje y textos & $41(57,7 \%)$ & $8(11,3 \%)$ & $1(1,4 \%)$ & $6(8,5 \%)$ & $15(21,1 \%)$ & 71 \\
\hline & Porta Linguarum & $13(17,6 \%)$ & $49(66,2 \%)$ & $1(1,4 \%)$ & $1(1,4 \%)$ & $10(13,5 \%)$ & 74 \\
\hline \multicolumn{2}{|r|}{ Total } & $82(42,5 \%)$ & $68(35,2 \%)$ & $4(2,1 \%)$ & $9(4,7 \%)$ & $30(15,5 \%)$ & $193(100 \%)$ \\
\hline
\end{tabular}

De los datos obtenidos se observa que en Porta Linguarum predominan las investigaciones sobre el inglés, puesto que es una revista especializada en didáctica de las lenguas extranjeras.

\section{Conclusiones}

Nuestro estudio representa un estudio bibliométrico sobre el estado actual de la investigación en Didáctica de la Lengua y la Literatura, en el que hemos manejado variables cualitativas en un análisis estadístico cuantitativo: análisis descriptivo de frecuencia y análisis inferencial. De esta manera hemos obtenido un diagnóstico de la situación: conocemos las tendencias existentes y las que predominan. El tema que predomina en la investigación es el de las habilidades lingüísticas (comprensión oral y escrita; y expresión oral y escrita), seguido por cualquier tema de literatura y en tercer lugar, de didáctica general. Se han puesto de manifiesto las lagunas también: por ejemplo, en las tres revistas son escasas las investigaciones sobre temas relacionados con la morfosintaxis, la ortografía, la fonética, el Espacio Europeo de Educación Superior, etc.

En cuanto al número de autores, predominan los artículos con un autor, pero se observa la tendencia que a mayor número de autores corresponden mayor número de investigaciones cuantitativas. Quizás se debe al hecho de que al reunir los conocimientos y los esfuerzos de varias personas, se puede afrontar mejor este tipo de investigación, porque es bastante laborioso. Se puede concluir que el trabajo en equipo favorece la investigación que utiliza métodos estadísticos.

Por otra parte, el uso de métodos estadísticos predomina en las revistas que tienen mejor posición en cuanto a los indicios de calidad. Entre las tres revistas analizadas Porta Linguarum es la única recogida en el JCR (Journal Citation Report) y el SSCI (Social Science Citation Index). Es también la revista con mayor factor de impacto. Por ello, conviene hacer hincapié en algunos datos por los que difiere de las demás revistas analizadas. En Porta Linguarum predomina el uso de métodos cuantitativos. Es la revista con mayor índice de internacionalidad de las contribuciones. Predomina el idioma inglés como lengua de publicación y como lengua investigada que es un factor más que contribuye a la mayor difusión de la revista. 
De los resultados obtenidos queremos resaltar que en la actualidad se nos reclama un cambio en la metodología de las investigaciones. La relación entre los indicios de calidad de las revistas y el método de investigación usado en los artículos publicados nos confirma que actualmente se valoran más las investigaciones basadas en análisis estadísticos cuantitativos o cualitativos frente a las investigaciones teóricas que no aplican análisis de datos ni resultados.

Sin embargo, el método de investigación teórica o revisión bibliográfica sin análisis de datos sigue predominando en el área de Didáctica de la Lengua y la Literatura. Consideramos que esto se debe a la insuficiente formación en este sentido de los profesores universitarios de esta área que son los principales investigadores. Las titulaciones de filología hacían hincapié en la tradición lingüística y literaria, sin incluir investigaciones empíricas ni ofrecer este tipo de formación: metodología de la investigación. Algunos investigadores han conseguido rectificar esta carencia durante sus estudios de tercer ciclo, pero tampoco ha sido accesible en esta etapa para la mayoría de los filólogos. Por consiguiente, consideramos que el profesorado necesita reciclarse para responder a la demanda actual en el ámbito de la investigación y si esta formación se gestionara desde sus empresas (universidades), participaría mayor número de investigadores. Como consecuencia, un mayor número de revistas del área aumentarían sus índices de calidad y, con ello, incrementaría la calidad de la productividad científica en el área de Didáctica de la Lengua y la Literatura.

\section{Bibliografía}

Alario Trigueros, A., Barrio Valencia, J., Calleja Largo, I., Castro Prieto, P., Castro del Val, C., Garrán Antolínez, M., Guillén Díaz, C. y Puertas, M. (1998). "El desarrollo del área de Didáctica de la Lengua y la Literatura: consideraciones sobre su consolidación", en Didáctica, vol.10: 161-178.

Battaner Arias, M. P. (2002). "Investigación en "Didáctica de la lengua y la literatura" y la consolidación del Área de Conocimiento", en Revista de Educación, vol. 328: 59-80.

Bausela Herreras (2010). Recensión del libro Para una transformación de la universidad. Nuevas relaciones entre investigación, saber y docencia, en Revista Docencia e Investigación, 10: 329-331.

Camps, A. (2004). "Objeto, modalidades y ámbitos de la investigación en Didáctica de la Lengua", en Lenguaje, vol. 32: 7-27.

Escobar Urmeneta, C. y Sánchez Sola, A. (2009). "Language Learning through tasks in a content and language integrated learning (CLIL) science classroom", en Porta Linguarum, 1: $65-83$.

Fernández Cano, A. y Bueno Sánchez, Á. (1998). "Síntesis de estudios bibliométricos españoles en educación. Una dimensión evaluativa", en Revista española de documentación científica, vol. 21, (3): 269-285.

Fernández-Quijada, D. (2010). "El perfil de las revistas españolas de comunicación (2007-2008)", en Revista española de documentación científica, vol. 33 (4): 553-581.

García-Heras Muñoz, A. (2007). "Programas informáticos de corrección gramatical en el aprendizaje de una lengua extranjera (inglés): expresión escrita", en Docencia e investigación: revista de la Escuela Universitaria de Magisterio de Toledo, 17: 71-101. 
Makkey (1965). Language Teaching Analysis. London: Longman. Citado por Vez Jeremías (2009).

Níkleva, D. G., Cortina Pérez, B. (2011). "Tendencias en la investigación actual en Didáctica de la Lengua y la Literatura”, en Núñez Delgado y Rienda Polo (eds.), La investigación en Didáctica de la Lengua y la Literatura: situación actual y perspectivas de futuro. Madrid: SEDLL (Sociedad Española de Didáctica de la Lengua y la Literatura), 2415-2428.

Sancho, J. M. (2001). "Docencia e investigación: una profesión, dos mundos", en Educar, 28: 41-60. Citado por Bausela Herreras (2010).

Vez Jeremías, J. M. (2009). "Ámbitos de la investigación española en Didáctica de las LenguasCulturas: estudio de un caso", en Lenguaje y textos, vol. 29, 25-45.

Vidal, J. y Quintanilla, M. A. (2000). "The teaching and research relationships within institutional evaluation”, en Higher Education, 40: 221-229. Citado por Bausela Herreras (2010). 